\title{
EMBOLIZAÇÃO ARTERIAL PARA TRATAMENTO DE MIOMA UTERINO: UMA REVISÃO DA LITERATURA
}

\section{ARTERIAL EMBOLIZATION TO TREAT UTERINE MYOMA: A LITERATURE REVIEW}

\author{
Suelen dos Santos HENRIQUE ${ }^{1 *}$, André João Rodrigues Espelho ROSSI ${ }^{2}$, Camila \\ Rocha SCHNECKENBERG ${ }^{2}$, Luana LOPES ${ }^{2}$, Marcia Regina RIBEIRO' ${ }^{2}$, Ana \\ Claudia Garabeli Cavalli KLUTHCOVSKY ${ }^{3}$, Ricardo Zanetti GOMES ${ }^{4}$ \\ ${ }^{1 *}$ Autor para contato: Acadêmica do Curso de Medicina. Universidade Estadual de Ponta \\ Grossa (UEPG). Avenida Carlos Cavalcanti, 4748, Uvaranas, CEP 84030-900, Ponta Grossa, \\ PR, Brasil. E-mail: suelenhenrique@hotmail.com \\ ${ }^{2}$ Acadêmicos do Curso de Medicina. Universidade Estadual de Ponta Grossa (UEPG). Ave- \\ nida Carlos Cavalcanti, 4748, Uvaranas, CEP 84030-900, Ponta Grossa, PR, Brasil. \\ ${ }^{3}$ Professora Adjunta de Práticas de Saúde I. Departamento de Medicina. Universidade \\ Estadual de Ponta (UEPG). Avenida Carlos Cavalcanti, 4748, Uvaranas, 84030-900, Ponta \\ Grossa, PR, Brasil. \\ ${ }^{4}$ Professor Adjunto de Cardiologia \& Vascular. Departamento de Medicina. Universidade \\ Estadual de Ponta (UEPG). Avenida Carlos Cavalcanti, 4748, Uvaranas, 84030-900, Ponta \\ Grossa, PR, Brasil.
}

\section{RESUMO}

Miomas uterinos podem causar hemorragia intensa, dores e reduzida probabilidade de gravidez. A histerectomia ou miomectomia tem sido tradicionalmente a principal opção de tratamento, mas com risco de complicações. A embolização da artéria uterina é uma opção de tratamento mais recente. Este estudo objetivou fazer uma revisão sistemática da literatura até junho de 2015, sobre embolização arterial de mioma uterino, em estudos brasileiros, nas bases de dados SciELO e MEDLINE. Um total de 11 artigos foram identificados, todos da Região Sudeste. As 454 mulheres incluídas nos estudos tinham idade entre 22 a 56 anos. As reduções no volume médio do útero após a embolização da artéria uterina (variação de $52 \%$ a $27,4 \%$ ) e/ou reduções do mioma predominante (variação de 40,5\% a 22\%) foram comparáveis à literatura internacional. Observou-se redução dos sintomas, melhora da qualidade de vida, preservação da função ovariana e, para aquelas submetidas à miomectomia posterior, diminuição do sangramento operatório, incisões menores e maior preservação do útero. Outros achados incluíram redução qualiquantitativa do colágeno nos miomas, redução da absorção de radiação pelo ovário e pele nas pacientes em novo protocolo de arteriografia e aumento do sinal endometrial nas imagens ponderadas em T2 e pós-contraste. Em 15 gestações pós-embolização, houve 14 nascimentos (um gemelar) e dois abortamentos precoces. Sintomas ou complicações após a embolização foram relatados. Em geral, o procedimento apresentou bons resultados nos estudos brasileiros. Como técnica relativamente recente, seria interessante a promoção de mais estudos com acompanhamento da evolução das pacientes em longo prazo.

Palavras-chave: Embolização da Artéria Uterina; Mioma; Útero; Revisão.

\begin{abstract}
Uterine myoma may cause heavy bleeding, pain and reduced likelihood of pregnancy. Hysterectomy or myomectomy has traditionally been the main treatment option but it carries a risk of complications. Uterine artery embolization is a newer treatment option. This is a systematic review of the literature on arterial embolization of uterine myoma in Brazilian studies, in the databases SciELO and MEDLINE, up to June 2015. A total of
\end{abstract}


11 papers were identified, all from the Southeast. The 454 women included in the study were aged between 22-56 years old. Reductions in the average volume of the uterus after uterine artery embolization (range $52 \%$ to $27.4 \%$ ) and/or reductions in the dominant uterine myoma (range $40.5 \%$ to $22 \%$ ) were comparable to the literature. There were reduction of symptoms, improvement in quality of life, preservation of ovarian function and, for those undergoing myomectomy later, reduced operative bleeding, smaller incisions and greater preservation of the uterus. Other findings included qualitative and quantitative reduction of collagen in uterine myoma; reduced absorption of radiation by the ovary and skin in patients in the new arteriography protocol and increased endometrial signal in T2-weighted images and post-contrast. In 15 post-embolization pregnancies there were 14 live births (one twin) and two early miscarriages. Symptoms or complications have been reported after embolization. In general, the procedure had good results in Brazilian studies. As a relatively new technique, it would be interesting to promote further studies on monitoring changes in patients on long term.

Keywords: Uterine Artery Embolization; Myoma; Uterus; Review

\section{INTRODUÇÃO}

O mioma uterino é o tumor benigno mais comum que pode ocorrer no sistema reprodutor feminino (SU et al., 2012). A doença ocorre em cerca de vinte por cento das mulheres em idade reprodutiva (DUHAN, 2013), sendo mais comum entre 30 e 50 e em mulheres de ascendência afro-caribenha do que em mulheres caucasianas. Fatores de risco para miomas incluem: idade, nuliparidade, raça, história familiar e obesidade. Em dois terços dos casos, não há sintomas. Contudo, tumores maiores podem causar hemorragia, pressão anormal sobre a bexiga ou intestino e a paciente pode ter dificuldade em engravidar (KING; OVERTON, 2011).

A história clínica e métodos de palpação na região pélvica continuam sendo importantes no diagnóstico dos miomas (SU et al., 2012). Miomas são frequentemente descobertos como um achado incidental no ultra-som, mas também podem apresentar-se nas seguintes formas: sangramento uterino anormal e menorragia, infertilidade, massa pélvica, aumento abdominal, sintomas de pressão (frequência e/ou constipação urinária), retenção urinária ou dor pélvica aguda devido à torção de um mioma pedunculado. Durante a gravidez, o mioma pode crescer e sofrer degeneração causando dor (KING; OVERTON, 2011). Para concluir, devem-se acrescentar técnicas de imagens, principalmente a ultrassonografia transvaginal. Outros exames que podem ser usados são ultrassonografia com Doppler, tomografia computadorizada, ressonância magnética ou tomografia por emissão de pósitrons, embora tais técnicas sejam mais caras e menos utilizadas (SU et al., 2012).
O tratamento do mioma inclui medicamentos que devem ser usados para melhorar os sintomas e/ ou reduzir os miomas antes da cirurgia. As mulheres com miomas maiores de $3 \mathrm{~cm}$ de diâmetro, causando sintomas significativos, dor ou pressão e que pretendam manter o útero, podem considerar a miomectomia. A histerectomia é o tratamento padrão para mulheres com miomas sintomáticos que não melhoraram com o tratamento clínico. Caso a mulher não deseje mais ter filhos e os miomas sejam múltiplos, histerectomia fornece a cura permanente (DOHERTY et al., 2014; KING; OVERTON, 2011).

Apesar de o tratamento cirúrgico ser o método tradicional para tratamento do mioma, tanto a histerectomia como a miomectomia podem ter complicações (GUPTA et al., 2012; METTLER et al., 2012), não sendo a histerectomia uma opção para aquelas pacientes com desejo de manter a fertilidade e o útero.

A embolização da artéria uterina (EAU), técnica utilizada desde os anos 1990 (THE PRACTICE COMMITTEE, 2008), é uma opção de tratamento que bloqueia o fornecimento de sangue para o útero e, assim, diminui os miomas e reduz os seus efeitos. É oferecida como uma terapia alternativa conservadora eficaz e segura para o tratamento de miomas em mulheres que não desejam engravidar (GUPTA et al., 2012). Desde o início de sua utilização, tem sido progressivamente mais aceita como um procedimento minimamente invasivo e que preserva o útero, sendo cada vez mais realizado, principalmente nos Estados Unidos e na Europa Ocidental (COMMITTEE ON GYNECOLOGIC PRACTICE, 2004). 
Apesar de a EAU ser uma técnica relativamente recente, vários relatos e revisões sobre essa nova opção de tratamento de mioma uterino vêm sendo descritos nos últimos anos em diversos países. Como essa técnica tem sido utilizada no Brasil, o objetivo deste trabalho foi realizar uma revisão sistemática da literatura sobre embolização arterial de mioma uterino, com base em estudos brasileiros publicados.

\section{MATERIAL E MÉTODO}

Trata-se de um estudo de revisão sistemática da literatura sobre tratamento de mioma uterino utilizando-se a técnica de embolização arterial, realizados no Brasil. A pesquisa bibliográfica é uma fonte ímpar de informações, contribuindo com muitas formas do saber, como a atividade intelectual e o conhecimento cultural. Ocupando lugar de destaque dentre as demais, ela é um conjunto de conhecimentos das mais variadas obras, que proporciona ao leitor conhecimentos para pesquisas futuras (FACHIN, 2006).

Para o levantamento, realizado no dia 30 de junho de 2015, foram utilizados os bancos de dados da Biblioteca Científica Eletrônica Online (SciELO) e do Medical Literature Analysis and Retrieval System Online (MEDLINE). A SciELO contém dados desde 1997 (data de início da coleta de dados do sistema). Escolheu-se essa base de dados por abranger uma coleção selecionada de periódicos científicos brasileiros, de acesso livre e com textos originais na íntegra. A busca foi realizada utilizando-se os descritores "mioma", "fibromioma" e "leiomioma" no campo de busca "assunto" e no campo "todos os índices", um de cada vez, juntamente ao descritor "embolização" no campo de busca "assunto" e no campo "todos os índices", sem restrição de período ou de idioma. A escolha dos melhores termos a serem utilizados na estratégia de busca foi feita por uma pesquisa cuidadosa no vocabulário dos Descritores em Ciências da Saúde (DeCS) da Biblioteca Virtual em Saúde (BVS, 2014).

A estratégia de busca seguiu a metodologia que envolve as seguintes etapas: inclusão de termos relacionados à condição (mioma uterino) e inserção de termos relacionados à intervenção (embolização). A observância desses passos conferiu aumento de sensibilidade a esta revisão. Os trabalhos de interesse foram selecionados mediante a leitura do título e do resumo. Excluindo-se as repetições, um artigo de revisão e um relato de casos, foram selecionados 11 artigos, publica- dos entre 2001 e 2013, que tratavam especificamente de estudos realizados no Brasil sobre embolização para tratamento de mioma uterino.

Para a busca de estudos brasileiros publicados em periódicos de outros países, foi utilizado o banco de dados do MEDLINE. Trata-se de uma base de dados em nível mundial, de acesso gratuito pela internet, da National Library of Medicine e do NIH do Departamento de Saúde e Serviços Humanos do governo norte-americano. Para o MEDLINE, os termos de busca utilizados foram: "myoma", "fibroids", "embolization" e "Brazil". Após excluir as repetições dos artigos que já haviam sido selecionados na base SciELO e de um relato de caso, nenhum artigo foi selecionado.

Os 11 artigos foram então quantificados e analisados segundo as seguintes variáveis: ano de publicação, periódico, local da realização da pesquisa, objetivos, número e idade dos sujeitos pesquisados, grupo-controle, resultados e sintomas ou complicações após o procedimento. Os dados foram tratados por meio da análise das frequências absoluta e relativa.

\section{RESULTADOS E DISCUSSÃO}

Foram analisados 11 estudos realizados no Brasil sobre o tema "embolização arterial de miomas uterinos”. Todos foram publicados em revistas médicas, havendo um predomínio dos periódicos da Revista Brasileira de Ginecologia e Obstetrícia, com cinco publicações (45,5\%) e Radiologia Brasileira, com duas $(18,2 \%)$. Os demais periódicos com publicações foram: São Paulo Medical Journal, Clinics, Revista do Colégio Brasileiro de Cirurgiões e Einstein, cada um com um artigo publicado.

A tabela 1 apresenta os dados dos artigos nacionais $(\mathrm{n}=11)$ sobre embolização arterial de mioma uterino quanto aos autores, ano de publicação, local do estudo, objetivos, número de sujeitos pesquisados, idade dos participantes, grupo-controle, resultados e sintomas ou complicações após a EAU.

Os artigos foram publicados entre os anos de 2001 e 2013. Três artigos (27,3\%) foram publicados entre os anos de 2001 e 2003 e 8 (72,7\%) entre 2007 e 2013, revelando, assim, uma tendência de aumento no número de publicações nos últimos anos. Observouse que todos os trabalhos foram realizados na região Sudeste, sendo $9(81,8 \%)$ realizados na cidade de São Paulo e 2 (18,2\%) deles no Rio de Janeiro. 
A maior parte dos artigos $(n=7,63,6 \%)$ objetivou, dentre outras variáveis, avaliar a redução do volume médio dos miomas uterinos e/ou do mioma dominante após a EAU, objetivando-se em um deles testar uma nova substância para a embolização. Outros objetivos dos estudos foram: comparação dos sintomas, da qualidade de vida, da função ovariana e das repercussões na morfologia do tecido uterino, todos antes e após a EAU. Um estudo quantificou a radiação ionizante absorvida pelo ovário e pele em pacientes submetidas à EAU e sugeriu um protocolo para reduzir riscos do procedimento; e outro avaliou os resultados da gravidez, complicações e resultados neonatais em mulheres anteriormente submetidas à EAU.

Um total de 454 mulheres foram incluídas nos 11 estudos, variando de 12 a 100 mulheres. Sete artigos $(63,6 \%)$ pesquisaram mulheres com idade que variavam de 22 a 50 anos e $2(8,2 \%)$ incluíram também mulheres com mais de 50 anos. Um artigo relatou idade média das pacientes de 36,7 anos e outro não especificou a idade das participantes. Em três estudos (27,3\%), foi mencionado grupo controle para comparação dos resultados. Em todos os trabalhos brasileiros publicados, a técnica utilizada na embolização das artérias uterinas foi por acesso da artéria femoral direita.

A redução no volume médio do útero e/ou do mioma dominante após a EAU foram as variáveis mais pesquisadas nos artigos brasileiros $(n=7$, 63,6\%). As reduções médias do volume uterino após EAU variaram de $52 \%$ a $27,4 \%$, sendo encontradas 52\% (KISILEVSTZ; MARTINS, 2003), 42,9\% (NASSER et al., 2010), 41,6\% (SENA- MARTINS et al., 2003), 35\% (BERNARDO et al., 2011), 32,5\% (KISILEVSKY, 2007), 32,3\% (GHIARONI et al., 2013) e 27,4\% (MESSINA et al.). As reduções médias dos volumes dos miomas dominantes após a EAU foram de 40,5\% (SENA MARTINS et al., 2003), 31,1\% (GHIARONI et al., 2013) e 22\% (BERNARDO et al., 2011). Esses valores são comparáveis aos encontrados em estudos internacionais (MARA et al., 2012; CHOI et al., 2013). Bonduki et al. (2009) observaram uma redução quantitativa e qualitativa significativa do colágeno nas lâminas histológicas dos miomas após EAU, comprovando redução da massa tumoral.

Também foi demonstrado, após a realização da EAU, redução dos sintomas das pacientes em seis estudos (54,5\%) (GHIARONI et al., 2013; KISILEVSKY; MARTINS, 2003; KISILEVSKY, 2007; MESSINA et al., 2001; NASSER et al., 2010; SENA-MARTINS et al., 2003), melhora da qualidade de vida em um estudo (9,1\%) (KISILEVSKY, 2007) assim como o não aumento dos níveis de FSH (BERNARDO et al., 2011).

Ghiaroni et al. (2013) avaliaram a utilização de uma nova partícula de polivinil álcool e polivinil acetato esférica para EAU em pacientes com miomas e indicação cirúrgica. Além da redução média do volume uterino e do maior mioma, houve melhora na concentração da hemoglobina, dos sinais e sintomas e diminuição do sangramento operatório, incisões menores e maior chance de preservação do útero durante a miomectomia.

Assim, de modo geral, observou-se nesses estudos uma redução significativa do tamanho médio do útero e/ou do mioma dominante, além da redução nos sintomas associados à doença. Em uma meta-análise sobre avaliação da eficácia e da segurança da EAU em relação à miomectomia, com 680 pacientes em oito ensaios, observou-se que os procedimentos cirúrgicos para o tratamento de miomas uterinos resultaram mais eficazes do que EAU. Contudo, o risco relativo entre pacientes submetidas à EAU e o grupo-controle submetido à miomectomia foi de 0,95 (IC 95\% 0,92-0,99), com uma taxa de sucesso de $95 \%$ do grupo controle (LI; WEN, 2006).

Três estudos (27,3\%) analisados nesta revisão tiveram objetivos diferentes dos já citados anteriormente. Um deles encontrou redução significativa da absorção de radiação absorvida pelo ovário e pela pele nas pacientes submetidas à EAU em que se utilizou modificação do número de quadros por segundo nas aquisições arteriográficas, seguindo uma implantação rotineira de normas de proteção radiológica, comparadas com pacientes que receberam radiação contínua (AFFONSO et al., 2010).

Outro estudo brasileiro avaliou os resultados da gravidez, complicações e resultados neonatais em mulheres que já haviam sido submetidas à EAU. Das 187 mulheres que se submeteram a EAU, 75 tinham tentado engravidar em algum momento e 15 engravidaram pelo menos uma vez. Houve 2 abortos e 14 nasceram com sucesso (um gemelar). $\mathrm{O}$ tempo médio entre a embolização e a concepção foi de 23,8 meses. Um recém-nascido foi considerado com baixo peso ao nascer $(2,260 \mathrm{~kg})$. Todas as pacientes foram submetidas à cesariana. Existiram dois casos de placenta acreta (tratado com histerectomia em um caso), um 
Tabela 1 - Estudos brasileiros publicados sobre embolização arterial do útero (EAU) para tratamento de mioma uterino

(continua)

\begin{tabular}{|c|c|c|c|c|c|c|}
\hline $\begin{array}{l}\text { Autores, ano } \\
\text { de publicação } \\
\text { e local }\end{array}$ & Objetivos & $\begin{array}{l}\text { Sujeitos } \\
\text { (número) }\end{array}$ & $\begin{array}{l}\text { Idade } \\
\text { (anos) }\end{array}$ & $\begin{array}{c}\text { Grupo } \\
\text { Controle }\end{array}$ & Resultados & $\begin{array}{c}\text { Sintomas ou } \\
\text { complicações pós } \\
\text { EAU }\end{array}$ \\
\hline $\begin{array}{l}\text { Messina } \\
\text { et al. } \\
\text { (2001) } \\
\text { São Paulo-SP } \\
\end{array}$ & $\begin{array}{l}\text { Avaliar a involução dos } \\
\text { miomas uterinos após } \\
\text { EAU. }\end{array}$ & 18 & 35 a 50 & Sim & $\begin{array}{l}\text { Controle do sangramento ( } 86 \% \\
\text { dos casos), dismenorreia }(60 \%) \\
\text { e redução média de } 27,4 \% \text { no } \\
\text { volume uterino } 3 \text { meses pós } \\
\text { EAU. }\end{array}$ & $\begin{array}{l}\text { Três casos de } \\
\text { insuficiência ovariana } \\
\text { clínica e laboratorial. }\end{array}$ \\
\hline $\begin{array}{l}\text { Sena-Martins } \\
\text { et al. } \\
(\mathbf{2 0 0 3 )} \\
\text { São Paulo - SP }\end{array}$ & \begin{tabular}{|l|} 
Comparação dos \\
sintomas, do volume \\
médio do útero e do \\
mioma dominante antes e \\
12 semanas após EAU. \\
\end{tabular} & 32 & 27 a 51 & Não & $\begin{array}{l}\text { Redução significativa dos } \\
\text { sintomas, do volume médio } \\
\text { do útero }(41,6 \%) \text { e do mioma } \\
\text { dominante }(40,5 \%) \text {. }\end{array}$ & $\begin{array}{l}\text { Dor (100\%), fadiga } \\
(34 \%), \text { degeneração } \\
(3 \%), \text { eliminação } \\
\text { de mioma (6\%), } \\
\text { hematoma (9\%). }\end{array}$ \\
\hline $\begin{array}{l}\text { Kisilevzky et } \\
\text { al. (2003) } \\
\text { São Paulo - SP }\end{array}$ & $\begin{array}{l}\text { Resultados de uma } \\
\text { experiência clínica de } \\
\text { mulheres submetidas à } \\
\text { EAU para tratamento de } \\
\text { miomatose uterina. }\end{array}$ & 100 & 24 a 56 & Não & $\begin{array}{l}\text { Na reavaliação de } 76 \text { pacientes, } \\
\text { houve melhora dos sintomas em } \\
\text { mais de } 90 \% \text { dos casos e redução } \\
\text { média do volume uterino de } 52 \% \text {. }\end{array}$ & $\begin{array}{l}\text { Síndrome pós- } \\
\text { embolização: dor } \\
(95 \text { casos), náuseas } \\
\text { e/ou vômitos (42) e } \\
\text { indisposição geral } \\
\text { (34). }\end{array}$ \\
\hline $\begin{array}{l}\text {.Kisilevzky } \\
\text { (2007) } \\
\text { São Paulo - SP }\end{array}$ & $\begin{array}{l}\text { Avaliação dos sintomas, } \\
\text { qualidade de vida e } \\
\text { volume uterino antes e } \\
\text { após a EAU. }\end{array}$ & 40 & 22 a 46 & Não & $\begin{array}{l}\text { Melhora nos escores dos } \\
\text { sintomas de } 67,1 \% \text {, melhora na } \\
\text { qualidade de vida de } 52,6 \% \text { e } \\
\text { redução média do volume uterino } \\
\text { em } 32,5 \% \text { ) pós EAU. }\end{array}$ & Não relatado. \\
\hline $\begin{array}{l}\text { Bonduki } \\
\text { et al. (2009) } \\
\text { São Paulo - SP }\end{array}$ & $\begin{array}{l}\text { Analisar as repercussões } \\
\text { da EAU na morfologia } \\
\text { do tecido uterino, pela } \\
\text { quantificação de tecido } \\
\text { colágeno antes e após o } \\
\text { procedimento. }\end{array}$ & 15 & $\begin{array}{l}\text { Não } \\
\text { relatada }\end{array}$ & Não & $\begin{array}{l}\text { Redução quantitativa e } \\
\text { qualitativa significativa do } \\
\text { colágeno nas lâminas histológicas } \\
\text { dos miomas } 3 \text { meses após EAU, } \\
\text { comprovando redução da massa } \\
\text { tumoral. }\end{array}$ & Não relatado. \\
\hline $\begin{array}{l}\text { Affonso } \\
\text { et al. (2010) } \\
\text { São Paulo - SP }\end{array}$ & $\begin{array}{l}\text { Quantificar a radiação } \\
\text { ionizante absorvida } \\
\text { pelo ovário e pele em } \\
\text { pacientes submetidas } \\
\text { à EAU e sugerir um } \\
\text { protocolo para reduzir } \\
\text { riscos do procedimento. }\end{array}$ & 73 & 27 a 48 & Sim & $\begin{array}{l}\text { Redução da absorção de radiação } \\
\text { pelo ovário e pele nas pacientes } \\
\text { em que se utilizou modificação } \\
\text { do número de quadros por } \\
\text { segundo nas aquisições } \\
\text { arteriográficas da EAU. }\end{array}$ & Dor. \\
\hline $\begin{array}{l}\text { Nasser } \\
\text { et al. (2010) } \\
\text { São Paulo - SP }\end{array}$ & $\begin{array}{l}\text { Avaliar a evolução clínica } \\
\text { e redução do volume } \\
\text { de miomas uterinos } \\
\text { volumosos após EAU. }\end{array}$ & 26 & 27 a 45 & Não & $\begin{array}{l}\text { Melhora clínica em } 25 \text { pacientes } \\
(96 \%) \text {. Em } 15 \text { pacientes avaliadas } \\
\text { após } 6 \text { meses, e redução } \\
\text { volumétrica média uterina foi de } \\
42,9 \% \text {. }\end{array}$ & $\begin{array}{l}\text { Um caso de necrose } \\
\text { e expulsão parcial } \\
\text { do mioma (realizado } \\
\text { miomectomia). Outro } \\
\text { necessitou segunda } \\
\text { intervenção para um } \\
\text { resultado adequado. }\end{array}$ \\
\hline $\begin{array}{l}\text { Bonduki } \\
\text { et al. (2011) } \\
\text { São Paulo - SP }\end{array}$ & \begin{tabular}{|l|} 
Avaliar os resultados da \\
gravidez, complicações \\
e resultados neonatais de \\
187 mulheres submetidas \\
à EAU anterior
\end{tabular} & 15 & 26 a 40 & Não & $\begin{array}{l}\text { Das } 15 \text { gestantes, houve } 14 \\
\text { nascimentos com sucesso (um } \\
\text { gemelar) e dois abortamentos } \\
\text { precoces. }\end{array}$ & $\begin{array}{l}\text { Aumento do risco de } \\
\text { parto cesáreo. }\end{array}$ \\
\hline $\begin{array}{l}\text { Bernardo et al. } \\
(2011) \\
\text { Rio de Janeiro } \\
\text {-RJ }\end{array}$ & \begin{tabular}{|l|} 
Avaliar a redução de \\
volume uterino e a \\
função ovariana antes e 3 \\
meses após EAU.
\end{tabular} & 29 & $\begin{array}{l}\text { Média } \\
\text { de } 36,7\end{array}$ & Não & $\begin{array}{l}\text { Diminuição do volume uterino } \\
\text { em } 35 \% \text {, do diâmetro do mioma } \\
\text { dominante em } 22 \% \text { e não houve } \\
\text { aumento significativo dos níveis } \\
\text { de FSH (sem alteração na função } \\
\text { ovariana). }\end{array}$ & Não relatado. \\
\hline
\end{tabular}


Tabela 1 - Estudos brasileiros publicados sobre embolização arterial do útero (EAU) para tratamento de mioma uterino

(conclusão)

\begin{tabular}{|c|c|c|c|c|c|c|}
\hline $\begin{array}{c}\text { Autores, ano } \\
\text { de publicação } \\
\text { e local }\end{array}$ & Objetivos & $\begin{array}{l}\text { Sujeitos } \\
\text { (número) }\end{array}$ & $\begin{array}{l}\text { Idade } \\
\text { (anos) }\end{array}$ & $\begin{array}{c}\text { Grupo } \\
\text { Controle }\end{array}$ & Resultados & $\begin{array}{c}\text { Sintomas ou } \\
\text { complicações pós } \\
\text { EAU }\end{array}$ \\
\hline $\begin{array}{l}\text { Ghiaroni } \\
\text { et al. (2013) } \\
\text { Rio de Janeiro } \\
\text {-RJ }\end{array}$ & $\begin{array}{l}\text { Avaliar a utilização de } \\
\text { uma nova partícula de } \\
\text { polivinil álcool e polivinil } \\
\text { acetato esférica (PVA- } \\
\text { PVAc), para embolização } \\
\text { das artérias uterinas, em } \\
\text { pacientes portadoras de } \\
\text { mioma, com indicação } \\
\text { cirúrgica. }\end{array}$ & 12 & 23 a 44 & Não & $\begin{array}{l}\text { A EAU com partículas de PVA- } \\
\text { PVAc mostrou-se promissora } \\
\text { no preparo da cirurgia. Houve } \\
\text { redução média do volume } \\
\text { uterino }(32,3 \%) \text { e do maior } \\
\text { mioma }(31,1 \%) \text {, melhora na } \\
\text { concentração da hemoglobina e } \\
\text { dos sinais/sintomas. Observou- } \\
\text { se diminuição do sangramento } \\
\text { operatório, incisões menores e } \\
\text { maior preservação do útero. }\end{array}$ & Não relatado. \\
\hline $\begin{array}{l}\text { Jacobs } \\
\text { et al. (2013) } \\
\text { São Paulo - SP }\end{array}$ & $\begin{array}{l}\text { Avaliar as alterações } \\
\text { endometriais em } \\
\text { pacientes com mioma } \\
\text { uterino sintomático } \\
\text { pela ressonância de alto } \\
\text { campo antes e } 6 \text { meses } \\
\text { após EAU. }\end{array}$ & 94 & 27 a 48 & $\mathrm{Sim}$ & $\begin{array}{l}\text { Após a EAU houve } \\
\text { significativo aumento do sinal } \\
\text { endometrial nas imagens } \\
\text { ponderadas em T2 } \\
\text { e nas imagens pós-contraste, } \\
\text { inferindo possível edema e } \\
\text { aumento do fluxo endometrial. }\end{array}$ & Não relatado. \\
\hline
\end{tabular}

caso de ruptura prematura das membranas e um caso de pré-eclampsia. Houve maior risco para parto cesáreo, mas não houve grandes riscos obstétricos, sugerindo que a gravidez após a embolização arterial uterina é possível sem morbidade ou mortalidade (BONDUKI et al., 2011).

Recente revisão sobre a EAU na modificação do padrão de risco de gestantes portadoras de miomatose $(\mathrm{n}=227)$ analisou, além das taxas de aborto, outras cinco complicações tardias: parto prematuro, hemorragia pós-parto, cesariana, restrição de crescimento intrauterino e má apresentação. O resultado do estudo mostrou que mulheres com miomatose que foram submetidas à EAU apresentaram maiores taxas de aborto, possibilidade de parto cesáreo e hemorragia pós-parto. Esses resultados sugerem que a EAU é um procedimento de risco para mulheres que desejam engravidar(HOMER; SARIDOGAN, 2010). Portanto, a EAU não deveria ser recomendada para mulheres com mioma uterino que desejam manter ou aumentar sua fertilidade, já que a segurança e a efetividade do procedimento para essas mulheres ainda não estão bem estabelecidos (THE PRACTICE COMMITTEE, 2008).

Estudo comparativo de dois tipos diferentes de terapia oclusiva de mioma uterino foi realizado com 100 mulheres nos Emirados Árabes. As mulheres com mioma(s) sem indicação para miomectomia laparoscópica foram tratados com EAU ou oclusão laparoscópi- ca da artéria uterina. Ambos os métodos foram eficazes no tratamento de mulheres com desejo de gravidez e daquelas com miomas sem indicação para miomectomia laparoscópica. EAU foi mais eficaz em provocar uma isquemia completa dos miomas, mas esteve associado a maior risco de necrose intrauterina. Ambos os métodos apresentaram baixas taxas de complicações graves, exceto por uma alta taxa de aborto (MARA et al., 2012).

O último estudo brasileiro analisado evidenciou que, após a EAU, houve um significativo aumento do sinal em T2 (inferindo maior grau de hidratação ou processo inflamatório tecidual) e maior realce pós-contraste (inferindo maior afluxo sanguíneo) no endométrio. Esses achados podem abrir uma nova frente de estudos sobre as alterações endometriais relacionadas à EAU, pois seria possível, porém ainda não comprovado, que um endométrio mais vascularizado tenha maior chance de possibilitar uma gestação saudável (JACOBS et al., 2013).

Sintomas ou complicações após a EAU foram relatados em alguns estudos brasileiros, como dor (AFFONSO et al., 2010; KISILEVSKY; MARTINS, 2003; SENA-MARTINS et al., 2003), náuseas e/ou vômitos e indisposição geral (KISILEVSKY; MARTINS, 2003), fadiga, degeneração, eliminação de mioma e hematoma (SENA-MARTINS et al., 2003), três casos de insuficiência ovariana clínica e laboratorial (MESSINA 
et al., 2001), miomectomia por necrose e expulsão parcial do mioma e necessidade de segunda intervenção para um resultado adequado (NASSER et al., 2010) e aumento do risco de parto cesáreo em gestantes após EAU (BONDUKI et al., 2011).

Em 8.159 pacientes submetidas a EAU, oriundas de 54 estudos, as complicações principais que exigiram um tempo maior de internação foram constatadas em cerca de $2,9 \%$ das pacientes, incluindo amenorreia $(3,9 \%)$, expulsão do mioma $(4,7 \%)$ e histerectomia $(0,7 \%)$. Houve necessidade de repetição de 81 casos de EAU, 81 miomectomias e 257 histerectomias. Os resultados mostraram que a EAU se mostrou eficaz com baixas taxas de complicação (TOOR et al., 2012).

Resultados em longo prazo com base em registros de 2.112 pacientes submetidas a EAU por miomas sintomáticos demonstraram melhora na qualidade de vida e consequente necessidade de histerectomia, miomectomia ou embolização das artérias uterinas em $9,79 \%, 2,82 \%$ e $1,83 \%$ dos pacientes, respectivamente (GOODWIN et al., 2008).

Segundo Gupta et al. (2012), com relação à segurança, não houve diferenças nos principais índices de complicações, sendo a porcentagem de mulheres com complicações menores maior após EAU em comparação às submetidas à cirurgia. A embolização foi associada a uma maior taxa de pequenas complicações pós-processuais, como corrimento vaginal, hematoma pós-punção e síndrome pós embolização (dor, febre, náuseas e vômitos), bem como maiores taxas de readmissão após a alta em comparação com a histerectomia.

AEAU apresentou vantagens de curto prazo sobre a cirurgia. Em médio e longo prazo, os benefícios foram similares, com exceção de uma taxa de reintervenção maior após a EAU (VAN DER KOOIJ et al., 2011). Confirmando os estudos brasileiros e levando em consideração as diferenças metodológicas e técnicas entre os estudos, em comparação com a cirurgia, as pacientes submetidas à EAU apresentaram menos complicações graves (JUN et al., 2012; MARTIN et al., 2013) e melhora na maioria dos aspectos de qualidade de vida após 6 meses (JUN et al., 2012).

Apesar de não ter sido uma variável pesquisada nos estudos brasileiros, a embolização parece reduzir significativamente o tempo de internação hospitalar e proporcionar um retorno rápido às atividades rotineiras, quando comparada às cirurgias como histerectomia ou miomectomia (GUPTA et al., 2012; JUN et al.,
2012; VAN DER KOOIJ et al., 2011). Por outro lado, as chances de uma nova intervenção cirúrgica dentro de cinco anos foram significativamente maiores após a EAU, tornando os custos desse procedimento equivalentes aos da histerectomia (GUPTA et al., 2012).

\section{CONCLUSÕES}

A EAU é um tratamento de primeira linha para miomas sintomáticos, e é oferecido como uma terapia alternativa à histerectomia e à miomectomia. Trata-se de um procedimento minimamente invasivo, com um período de recuperação curto e com preservação do útero, sendo extremamente importante para pacientes que não queiram se expor a riscos cirúrgicos, tenham contraindicações ou que não desejam retirar o útero.

Foram analisados 11 artigos nacionais sobre o tema EAU, publicados entre os anos de 2001 e 2013, com aumento no número de publicações nos últimos anos, sendo todos realizados na região Sudeste, em São Paulo e Rio de Janeiro. Um total de 454 mulheres foram incluídas nos estudos, com idades entre 22 e 56 anos, a maioria sem grupo controle. Os objetivos mais frequentes foram avaliar a redução do volume médio dos miomas uterinos e/ou do mioma dominante e avaliação dos sintomas das pacientes antes e após a EAU.

De modo geral, os estudos brasileiros apresentaram bons resultados e apontaram redução significativa do volume médio do útero $(52 \%$ a $27,4 \%)$ e do mioma dominante $(40,5 \%$ a $22 \%)$ após o procedimento, comparáveis com a literatura internacional, além da redução dos sintomas das pacientes. Os sintomas ou complicações após a EAU foram relatados em alguns estudos, sendo destacada a dor após o procedimento. Outros menos frequentes incluíram náuseas e/ou vômitos, indisposição geral, fadiga, degeneração, eliminação de mioma, hematoma, insuficiência ovariana clínica, necessidade de segunda intervenção para um resultado adequado e aumento do risco de parto cesáreo em gestantes após EAU.

Sendo uma técnica relativamente recente no tratamento de mioma uterino, para enriquecer o banco de dados brasileiro sobre o assunto, seria interessante a promoção de mais estudos que trouxessem objetivos de amplo espectro, relacionados ao tempo e aos custos das internações, tempo de recuperação, complicações e impacto sobre a fertilidade, principalmente com acompanhamento da evolução das pacientes em longo prazo. 


\section{REFERÊNCIAS}

AFFONSO, B. B.; NASSER, F.; SILVA, S. G. J.; SILVA, M. C.; ZLOTNIK, E.; MESSINA, M. L.; BACARAT, E. C. Estratégias para redução da exposição de radiação ionizante em mulheres submetidas à embolização de miomas uterinos. Revista Brasileira de Ginecologia e Obstetrícia, v. 32, n. 2, p. 77-81, 2010.

BERNARDO, A.; GOMES, M. T. V.; CASTRO, R. A.; GIRÃO, M. J. B. C.; BONDUKI, C. E.; YOKOYAMA, C. A. Impacto da embolização arterial do leiomioma uterino no volume uterino, diâmetro do mioma dominante e na função ovariana. Revista Brasileira de Ginecologia e Obstetrícia, v. 33, n. 8, p. 201-206, 2011.

BONDUKI, C. E.; DORNELAS, G. O.; BERNARDO, A.; SIMÕES, M. J.; CASTRO, R. A.; GOMES, M. T. V.; GIRÃO, M. J. B. C. Avaliação da proporção de colágeno no tecido uterino antes e após tratamento do leiomioma uterino pela embolização arterial. Revista Brasileira de Ginecologia e Obstetrícia, v. 21, n. 12, p. 598-60, 2009.

BONDUKI, C. E.; FELDNER, P. C.; SILVA, J.; CASTRO, R. A.; SARTORI, M. G. F.; GIRÃO, M. J. B. Pregnancy after uterine arterial embolization. Clinics, v. 66, n. 5, p. 807-810, 2011.

BVS. Biblioteca Virtual em Saúde. Descritores em Ciências da Saúde (DeCS). 2014. Disponível em: <http:// www.bireme.br/php/index.php>. Acesso em: 24 abr. 2014.

CHOI, H. J.; JEON, G. S.; KIM, M.; LEE, J. T.; YOON, J. H. Is uterine artery embolization for patients with large myomas safe and effective? A retrospective comparative study in 323 patients. Journal of Vascular and Interventional Radiology, v. 24, n. 6, p. 772-778, 2013.

COMMITTEE ON GYNECOLOGIC PRACTICE. American College of Obstetricians and Gynecologists. ACOG Committee Opinion: uterine artery embolization. Obstetrics \& Gynecology, v. 103, p. 403-404, 2004.

DOHERTY, L.; MUTLU, L.; SINCLAIR, D.; TAYLOR, H. Uterine fibroids: clinical manifestations and contemporary management. Reproductive Sciences, v. 21, n. 9, p. 10671092, 2014.

DUHAN, N. Advances in management of uterine myomas. Frontiers in Bioscience (Elite Ed), v. 1, n. 5, p. 12-22, 2013.

FACHIN, O. Fundamentos de metodologia. São Paulo: Saraiva, 2006.

GHIARONI, J.; LOPEZ, G. E.; COUTINHO, JUNIOR, A. C.; SCHANAIDER, A. Uterine artery embolization with spherical pva-pvac particles as preparation for surgical resection of miyomas. Revista do Colégio Brasileiro de Cirurgiões, v. 40, n. 5, p. 386-391, 2013.

GOODWIN, S. C.; SPIES. J.; WORTHINGTON-KIRSCH, R.; PETERSON, E.; PRON, G.; LI, S.; MYERS, E. R.
Uterine artery embolization for treatment of leiomyomata: long-term outcomes from the FIBROID Registry. Obstetrics \& Gynecology, v. 11, n. 1, p. 2-33, 2008.

GUPTA, J. K.; SINHA, A. S.; LUMSDEN, M. A.; HICKEY, M. Uterine artery embolization for symptomatic uterine fibroids. Cochrane Database Systematic Reviews, v. 25, n. 1, CD005073, 2012.

HOMER, H.; SARIDOGAN, E. Uterine artery embolization for fibroids is associated with an increased risk of miscarriage. Fertility and sterility, v. 94, n. 1, p. 324-330, 2010.

JACOBS, M. A. P.; NASSER, F.; ZLOTNIK, E.; MESSINA, M. L.; BARONI, R. H. Alterações endometriais após embolização de leiomiomas uterinos avaliadas por ressonância magnética de alto campo (3 Tesla). Einstein, v. 11, n. 1, p. 58-62, 2013.

JUN, F.; YAMIN, L.; XINLI, X.; ZHE, L.; MIN, Z.; BO, Z.; WENLI, G. Uterine artery embolization versus surgery for symptomatic uterine fibroids: a randomized controlled trial and a meta-analysis of the literature. Archives of Gynecology and Obstetrics, v. 285, n. 5, p. 1407-1413, 2012.

KING, R.; OVERTON, C. Management of fibroids should be tailored to the patient. Practitioner, v. 255, n. 1738 , p. 19-23, 2011.

KISILEVZKY, N. H. Embolização da artéria uterina para tratamento de miomas sintomáticos: impacto na qualidade de vida. Radiologia Brasileira, v. 40, n. 5, p. 289-96, 2007.

KISILEVZKY, N. H.; MARTINS, M. S. Embolização uterina para tratamento de mioma sintomático. Experiência inicial e revisão de literatura. Radiologia Brasileira, v. 36, n. 3, p. 129-140, 2003.

LI, G. T.; WEN, T. R. Meta-analysis of uterine arterial embolization and myomectomy in treatment of uterine myoma. Zhonghua Fu Chan Ke Za Zhi, v. 41, n. 10, p. 697-700, 2006.

MARA, M.; KUBINOVA, K.; MASKOVA, J.; HORAK, P.; BELSAN, T.; KUZEL, D.

Uterine artery embolization versus laparoscopic uterine artery occlusion: the outcomes of a prospective, nonrandomized clinical trial. Cardiovascular and Interventional Radiology, v. 35, n. 5, p. 1041-1052, 2012.

MARTIN, J.; BHANOT, K.; ATHREYA, S. Complications and reinterventions in uterine artery embolization for symptomatic uterine fibroids: a literature review and metaanalysis. Cardiovascular and Interventional Radiology, v. 36, n. 2, p. 395-402, 2013.

MESSINA, M. L.; MARTINS, V. M.; FRATEZI, A. C.; HALBE, H. W.; BOZZINI, N.; RIBEIRO, S. C.; PINOTTI, J. A. Tratamento dos leiomiomas por embolização das artérias uterinas. Revista Brasileira de Ginecologia e Obstetrícia, v. 23, n. 9, p. 597-602, 2001. 
METTLER, L.; Schollmeyer, T.; Tinelli, A.; Malvasi, A.; Alkatout, I. Complications of Uterine Fibroids and Their Management, Surgical Management of Fibroids, Laparoscopy and Hysteroscopy versus Hysterectomy, Haemorrhage, Adhesions, and Complications. Obstetrics and Gynecology International, v. 2012, 2012:791248. DOI: 10.1155/2012/791248.

NASSER, F.; AFFONSO, B. B.; JESUS-SILVA, S. G.; COELHO, D. O.; ZLOTNIK, E.; MESSINA, M. L.; BARACAT, E. C. Embolização de mioma uterino em mulheres portadoras de miomas volumosos. Revista Brasileira de Ginecologia e Obstetrícia, v. 32, n. 11, p. 530-535, 2010.

SENA-MARTINS, M.; ROTELI-MARTINS, C. M.; TADINI, V.; DE SOUZA, G. A.; KISILEVZKY, N.; LAZAR JUNIOR, F. Uterine artery embolization for the treatment of symptomatic myomas in Brazilian women. São Paulo Medical Journal, v. 121, n. 5, p. 185-90, 2003.

SU, W. H.; LEE, W. L.; CHENG, M. H.; YEN, M. S.; CHAO, K. C.; WANG, P. H. Typical and atypical clinical presentation of uterine myomas. Journal of the Chinese Medical Association, v. 75, n. 10, p. 487-493, 2012.

THE PRACTICE COMMITTEE OF THE AMERICAN SOCIETY FOR REPRODUCTIVE MEDICINE. Myomas and reproductive function. Fertility and sterility, v. 90, n. 3, sup. 3, p. S125-130, 2008.

TOOR, S. S.; JABERI, A.; MACDONALD, D. B.; MCLNNES, M. D. F.; SCHWEITZER, M. E.; RASULI, P. Complication rates and effectiveness of uterine artery embolization in the treatment of symptomatic leiomyomas: a systematic review and meta-analysis. American Journal of Roentgenology, v. 199, p. 1153-1163, 2012.

VAN DER KOOIJ, S. M.; BIPAT, S.; HEHENKAMP, W. J.; ANKUM, W. M.; REEKERS, J, Á. Uterine artery embolization versus surgery in the treatment of symptomatic fibroids: a systematic review and metaanalysis. American Journal of Obstetrics and Gynecology, v. 205, n. 4, 2011. DOI: http://dx.doi.org/10.1016/j.ajog.2011.03.016. 